\title{
CONSIDERACIONES TEÓRICAS ACERCA DEL APEGO EN ADULTOS
}

Theoretical considerations about attachment in adults

Dorys Ortiz-Granja-Pontificia Universidad Católica del Ecuador*, Pamela Acosta-RodasPontificia Universidad Católica del Ecuador**, Dominique Rubio-Pontificia Universidad Católica del Ecuador***, Nancy Lepe-Martínez-Universidad Católica del Maule Ecuador****, Milenko Del Valle-Universidad de Antofagasta Chile*****, David CadenaPontificia Universidad Católica del Ecuador******, Edenia López-Pontificia Universidad Católica del Ecuador*******, Francisco Hinojosa-PontificiaUniversidad Católica del Ecuador********, Carlos Ramos Galarza-Pontificia Universidad Católica del Ecuador y Universidad Tecnológica Indoamérica de Ecuador*********

\begin{abstract}
Resumen
Se analizan las posibles repercusiones sobre procesos afectivos y cognitivos cuando se producen alteraciones en la conformación de un apego seguro, a lo largo del ciclo vital pero, particularmente, en la adultez joven debido a las áreas importantes de desempeño en esta edad, como son: la universidad, la formación de pareja y el trabajo. Es así que, en el artículo se realiza una revisión sobre aspectos teóricos del apego, sus implicaciones relacionales y su impacto en la construcción de modelos representacionales que determinan el devenir de las personas y sus relaciones. Se analizan también los tipos de apego y luego, se describen algunas investigaciones realizadas usando este marco teórico, así como también las diversas formas de evaluar el apego a través de escalas y otros instrumentos que han sido validados en Europa y Asia. Se concluye que existe un débil desarrollo de la parte psicométrica en Latinoamérica y se plantea la importancia y necesidad de ampliar esta área en el contexto ecuatoriano.
\end{abstract}

Palabras clave: apego, apego seguro, apego evitativo, apego ambivalente, escalas de apego.

\begin{abstract}
Possible impacts on cognitive and affective processes are analyzed when the building of secure attachment is disrupted throughout the life cycle but, mainly, during young adulthood. This is due to the significant areas of development during this life stage, such as university, mate searching and work. Therefore, this article reviews theoretical issues of attachment, its relational involvement and its impact on the building of representational patterns that determine the evolution of people and their relationships. Additionally, this study analyzes the types of attachment and describes relevant research with this theory framework, as well as various ways of assessing attachment through scales and other instruments validated in Europe and Asia. It is concluded that there is a weak development of the psychometric area in Latin America; therefore, it is important and necessary to expand this area in the Ecuadorian context. ies.
\end{abstract}

Keywords: attachment, secure attachment, avoidant attachment, ambivalent attachment, scales attachment.

\footnotetext{
* Psicóloga. ORCID: https://orcid.org/0000-0003-0617-0361

** Psicóloga. ORCID: https://orcid.org/0000-0002-0096-2168

*** Psicóloga. ORCID: https://orcid.org/0000-0002-3672-1841

**** Psicóloga. ORCID: https://orcid.org/0000-0003-3574-044X

****** Psicólogo. ORCID: https://orcid .org/0000-0003-3739-1001

******* Psicólogo. ORCID: https://orcid.org/0000-0003-3770-9833

******** Psicóloga. ORCID: https://orcid.org/0000-0002-3867-8242

******** Psicólogo. ORCID: https://orcid.org/0000-0002-6172-4315

********* Psicólogo. caramos@puce.edu.ec ORCID: https://orcid.org/0000-0001-5614-1994
} 


\section{INTRODUCCIÓN}

Este artículo presenta una revisión teórica sobre el constructo apego. En materia psicológica, la teoría del apego es uno de los aportes más importantes de los últimos tiempos, para comprender las relaciones de los seres humanos, el desarrollo de la personalidad y los modos de ejercer el rol de padres.

Además, se trata de una línea de investigación prolífica que ha generado innumerables trabajos, ya que el apego se ha relacionado con aspectos patológicos y también con elementos positivos del desarrollo humano. En cuanto al apego en adultos, resulta interesante evidenciar la manera en que las funciones del apego van cambiando a lo largo del ciclo vital, lo que genera modificaciones en la forma de relacionarse, de acuerdo al estilo de apego que se vaya asumiendo (Fraley, 2019).

Por lo tanto, realizar una revisión teórica sobre el apego permitirá, por un lado, poder evidenciar lo que se sabe del constructo y por otro lado, sentar algunas bases para la creación de instrumentos, que permitan medir este constructo, en ciertas poblaciones de interés, a través de escalas válidas y confiables, que se ajusten a su contexto cultural.

Para hacer esta revisión se usará una metodología deductiva en la cual se partirá de la revisión de la teoría del apego y sus tipos, para llegar al tema de las escalas de evaluación en ese orden.

\section{CONCEPTUALIZACIÓN DEL APEGO}

En las primeras etapas del ciclo vital, los seres humanos solo pueden sobrevivir con el apoyo de figuras protectoras que cumplen funciones de alimentación, calor o apoyo en situaciones difíciles o enfermedades (Casullo \& Fernández Liporace, 2005). Además, una de las principales necesidades, presente durante toda la vida, es formar parte de una comunidad y relacionarse con los iguales.

Tomando en consideración estas premisas, surge la teoría del apego (Brando, Valera, \& Zárate, 2008), como una base para comprender las relaciones humanas presentes y futuras y el desarrollo de la personalidad en los individuos, a lo largo de la vida.
Esta teoría permite explicar los efectos que tienen los vínculos de protección establecidos a temprana edad, en el desarrollo psíquico de la persona y asimismo, las consecuencias de su ausencia; por lo que se considera como uno de los planteamientos teóricos más sólidos en el campo de la investigación sobre el desarrollo emocional y social de una persona (Oliva, 2004).

Bowlby (1998) formula esta teoría, luego de observaciones hechas acerca de los vínculos familiares (Casullo \& Fernández Liporace, 2005); en su base, se encuentra la necesidad de los niños de sentir seguridad y describió el vínculo (Olza, 2008) como un "instinto biológico destinado a garantizar la supervivencia de los bebés; es un lazo que se establece entre el recién nacido y su madre, que cumple la función biológica de promover la protección, la supervivencia y, en última instancia, la adaptación" (p. 121).

Para Bowlby (Brando, Valera, \& Zárate, 2008) el apego es una conducta instintiva cuyo objetivo es facilitar la adaptación. La primera relación del niño, por lo general con la madre o un adulto significativo, constituye el pilar fundamental de su desarrollo psíquico, que luego se traduce en la base para establecer la estructura de las relaciones con el otro. Así, relaciones seguras pueden generar bienestar psicológico y, por el contrario, relaciones inseguras ocasionan dudas personales y efectos adversos en la relación con los demás (Fraley, 2019).

Esta teoría se relaciona con la regulación del afecto ya que sus estilos son una muestra de los mecanismos de intercambio de comportamientos, como respuesta a situaciones que producen ansiedad (Casullo \& Fernández Liporace, 2005). Lo que diferencia al apego de otro tipo de relaciones es que proporciona sentimientos de seguridad y pertenencia (Casullo \& Fernández Liporace, 2005), ofrece una 'base de operaciones segura' a partir de la cual, el niño puede explorar el mundo (Olza, 2008).

El establecimiento de estos cimientos es esencial para el desarrollo adecuado de la personalidad ya que contribuyen a la formación de una convicción íntima de valor y al desarrollo de la capacidad de tener relaciones afectivas próximas con otras personas para dar y recibir atención y cuidado cuando es necesario; 
su función también es distinta a la de las relaciones que proveen guía, satisfacción sexual, experiencias comunes, etc.

El apego implica un sistema altamente organizado conformado por varias conductas. Así, en los primeros años de vida, el niño usa el llanto y la sonrisa para mantener la cercanía física y emocional del cuidador; posteriormente, el lenguaje incide en este proceso al poder expresar aquello que existe en el pensamiento, para luego, finalmente, establecer una relación perdurable en la etapa adulta (Balbi, 2004).

Así, el sistema de apego se manifiesta a través de un delicado equilibrio entre 'conductas exploratorias', por un lado, y 'conductas de acercamiento' por otro. La mayor o menor oscilación entre estos dos polos tiene que ver con la percepción sobre la cercanía/lejanía del cuidador, los peligros potenciales que pueden aparecer en el ambiente y las necesidades experimentadas.

Las conductas de exploración y de acercamiento según Bowlby (Balbi, 2004), son tan visibles y se realizan sin siquiera la mediación de palabras que le llevó a señalar la hipótesis de que "la conducta de apego se organiza mediante un sistema de control dentro del sistema nervioso central" (p. 123); lo cual implica, necesariamente, que existen zonas cerebrales que regulan y manejan estas conductas y, por ende, el apego.

Uno de los sistemas de control es la memoria (Tulving, 1972) como depósito de las experiencias primarias del ser humano que, en el caso del bebé, no puede explicar con palabras porque no maneja aún el lenguaje oral y que permanecen en forma de conexiones a las cuales, la persona no tiene acceso consciente directo, pero se "pondrán en marcha" cuando ocurran eventos similares.

Entonces, claramente se sabe que esto impacta sobre el proceso de aprendizaje, ya que un estudiante adulto, por ejemplo, puede conocer bien el tema que tiene que exponer pero fracasa estrepitosamente, debido a la ansiedad que experimenta al pararse frente a sus compañeros, porque se desencadenan reacciones similares a las experimentadas en momentos de estrés en su infancia que le recuerdan experiencias de fracaso.
Además, del establecimiento de este sistema de control, la relación figura de apego-bebé contribuye a que el niño aprenda a mentalizar; es decir, use su experiencia relacional actual como futuro predictor de la conducta de las demás personas. Todos estos datos se procesan y guardan en la memoria del bebé y se muestran en forma de conductas o comportamientos que, incluso, puede que ni el mismo niño entienda pero que son reacciones primarias establecidas en base a conexiones anteriores al lenguaje.

Por ende, este modelo constituye una base que será de utilidad para que el sujeto interprete las acciones e intenciones de los demás y, así, dirija su conducta. La noción de quiénes son las figuras de apego, en dónde se las puede encontrar y qué se espera de ellas es un aspecto importante del modelo, debido a que incluye componentes afectivos y cognitivos.

Main (Barroso, 2014) expone que este modelo va más allá de dirigir los sentimientos y la conducta; interviene también en la memoria, la atención y otros procesos cognitivos, razón por la cual, es de gran utilidad para comprender el aprendizaje en jóvenes adultos, sobre cuyo procesamiento cognitivo se puede hipotetizar que se aproximan o alejan del conocimiento en función de sus patrones de apego.

Bowlby (Martínez \& Santelices, 2005) propone el término 'representaciones mentales' o 'modelos operantes' para estos esquemas construidos en la infancia. Se describen como expectativas sobre las personas y las relaciones, que integran experiencias pasadas y presentes y sus patrones tanto cognitivos como emocionales. Bowlby (Oliva, 2004) describe estos modelos como una representación mental de sí mismo y de las relaciones con otros, incluido aquí, el aspecto abstracto del aprendizaje.

Por lo tanto, la formación del apego, entendido como un vínculo emocional, es el aspecto más importante del desarrollo social en la infancia puesto que, a partir de esta primera construcción, la persona establece la forma en que se relaciona con los demás por el resto de la vida.

Estos 'modelos internalizados' (Balbi, 2004) se vuelven el mapa con el cual, las personas comprenden 
las conductas de los demás y organizan sus respuestas, en consecuencia. El mapa está determinado, en gran parte, por las estructuras mentales más básicas y primarias que influyen en el desempeño de funciones mentales superiores (Pinedo \& Santelices, 2006), dirigen las respuestas de afecto y conducta con las que los adultos responden a las necesidades de los niños que están a su cargo.

Los adultos desarrollan sus propios modelos internalizados de acuerdo con el cuidado que recibieron en la infancia por parte de sus cuidadores; estos patrones suelen mantenerse estables a lo largo de la vida; sin embargo, pueden sufrir modificaciones debido a nuevas experiencias y relaciones de calidad diferente con otras figuras significativas. Barroso (2014) agrega que estos modelos se hacen más complejos pues se reinterpretan, ajustan y remodelan a lo largo de la vida.

Oliva (2004) ha clasificado las posibles respuestas de las figuras de apego en tres: mostrar sensibilidad a las llamadas del niño y permitir la cercanía, lo cual crea un apego seguro; mostrar insensibilidad y no permitir el acceso del niño, crea un apego inseguro evitativo y atender y permitir el acceso de manera inconsistente e imprevisible genera apego inseguro ambivalente.

En cada caso, la madre (Salvador, 2013) funciona como una suerte de: "Córtex auxiliar que identifica y responde a las necesidades del niño, calmándolo e interviniendo en su bienestar (homeostasis)" (p.4). Así, la regulación interna comienza como una 'regulación biológica interactiva' para luego transformarse en una 'autorregulación biológica autónoma' (Salvador, 2013).

En otras palabras, cuando el niño ha sido bien cuidado y respondido en una relación de contacto pleno y sintonía con su mundo interno, aprende a 'cuidarse a sí mismo', porque ha internalizado al otro como un objeto constante y proveedor de buenos cuidados. Cuando no ha sido así, la persona mostrará el legado en su dificultad para calmarse o experimentándose sobrepasado por sus emociones. La regulación emocional es una de las capacidades más directamente correlacionadas con el funcionamiento adaptativo y la salud de un ser humano (p. 4).
También, es importante indicar que desde hace mucho tiempo ya, se conoce el efecto estresante que tiene la separación del bebé de su madre lo cual, incluso, puede alterar el desarrollo cerebral del recién nacido ya que produce gran cantidad de cortisol, que afecta el desarrollo de áreas vitales en el cerebro como el hipocampo y la amígdala y esto puede impactar en su desarrollo posterior (Flores, 2010).

Además de las respuestas más o menos adecuadas de la madre, el apego también se establece gracias a otras condiciones como los logros en el desarrollo, tanto cognitivo como emocional del niño, combinadas con las características asertivas de los adultos que lo rodean, como por ejemplo sus respuestas eficaces ante sus necesidades, lo que determinará la calidad del apego (Brando, Valera, \& Zárate, 2008).

Por lo tanto, el desarrollo de apego a través del ciclo vital genera un contexto confortable en el cual establecer nuevas relaciones y mantener cierto grado de bienestar psicológico, si se desarrolla apropiadamente. Lo contrario también es cierto, si el contexto es inadecuado, con mucho estrés y violencia, esto afectará la calidad del apego establecido.

Para sintetizar, se puede entender el apego como la búsqueda de proximidad de una figura particular, la misma que es utilizada como refugio en momentos difíciles y como base para la exploración del mundo. El niño y su figura de apego, ya sea su madre u otro adulto responsable, desarrollan este vínculo desde los primeros años de vida.

A medida que el niño se desarrolla, algunas funciones del apego van cambiando de los padres hacia los compañeros a quienes acuden más ante las dificultades que tienen en el día a día. Sin embargo, los padres permanecen todavía como la base segura necesaria para la exploración del mundo (Fraley, 2019).

Además, en la vida adulta, el comportamiento de apego se organiza o se asocia más a la pareja o a los amigos que a los padres; sin embargo, respecto al vínculo entre adultos, todavía hay cuestiones que no han sido resueltas: una de ellas es el tiempo necesario para establecer relaciones de apego y, también, saber 
si existe una jerarquía de apego que haga que cuando una persona sube en ella mientras otra baja. De igual manera, se ha hipotetizado una relación entre el apego y el aprendizaje, lo que también constituye una línea interesante de investigación futura.

\section{TIPOS DE APEGO}

Ainsworth encontró tres estilos de apego: seguro, inseguro evitativo e inseguro ambivalente (Oliva, 2004), se describen sus características principales.

Apego seguro: los niños consideran a la madre como base segura, se sienten a gusto en situaciones nuevas mientras ella está presente; exploran lo que les rodea y regresan a su lado, de vez en cuando. Aunque quizá se inquietan cuando la madre sale, los niños acuden a ella en cuanto la ven y buscan su contacto.

Este tipo de apego crea un nivel de homeostasis neurofisiológico cuya ausencia genera alteraciones en el sistema nervioso (Flores, 2010).

Los niños con apego seguro tienen menos dificultades en las relaciones humanas; tienden a ser más competentes en lo social y lo emocional (Olza, 2008) y se muestran más cooperativos, empáticos, interesados en aprender y seguros. Sus madres se describen como sensibles y disponibles ante las llamadas del bebé y sus necesidades (Oliva, 2004), responden de manera rápida y positiva a sus pedidos de atención, necesidades y deseos, con calidez y muestras de afecto; están al tanto de su estado de ánimo, toman en cuenta sus sentimientos, responden en las situaciones cara a cara y alimentan al niño cuando lo demanda.

Las madres sensibles y disponibles tienden a brindar el nivel apropiado de respuesta a las necesidades del hijo, lo que favorece el establecimiento de una sincronía interactiva (Armus, Duhalde, Oliver, \& Wosxoboinik, 2012). La madre es vista como una base segura que le permite al niño, explorar el mundo que le rodea, puesto que su responsividad cotidiana le da al niño, la seguridad y confianza de que está protegido, mostrando un patrón saludable de conductas de apego.
En la vida adulta, el apego seguro está presente en las personas que tuvieron y recuerdan una relación cordial y cariñosa con la madre, fueron criados en un ambiente de interés por satisfacer sus necesidades, son adultos con confianza y con altos niveles de satisfacción en sus relaciones íntimas (Medina, Rivera $\&$ Aguasvivas, 2016). Una persona con estilo de apego seguro, es capaz de establecer una dependencia mutua segura, de manera que sus relaciones son más estables y satisfactorias (Brando, Valera, \& Zárate, 2008).

Apego inseguro evitativo: los niños no buscan la cercanía de la madre, se separan de ella al estar en una situación nueva y van a explorar los juguetes y objetos inmediatamente. Cuando la madre se aleja no se muestran angustiados, tampoco se ven atemorizados en presencia de un extraño; cuando la madre vuelve parecen evitarla, como si les resultara indiferente su conducta. Al parecer un $20 \%$ de los niños de un año están en este caso.

Los niños y niñas con este tipo de apego, a edades mayores, muestran más dificultades en las relaciones, con comportamientos evitativos de acercamiento e implicación emocional, por lo que son menos competentes en lo social y lo emocional. Los niños se muestran inseguros y preocupados por la cercanía con su madre porque han comprendido que no cuentan con su apoyo, por lo que su indiferencia es una forma de reacción defensiva. Al haber sufrido rechazos a lo largo de la vida, estos niños negarán sus necesidades para evitar sentir frustración (Brando, Valera y Zárate, 2008).

Las madres de niños con apego inseguro evitativo, ignoran sus señales; así, el niño aprende, rápidamente, que su madre no está disponible cuando la necesite, por lo que deja de depender de ella y se vuelve más autosuficiente. Las madres de estos niños se han mostrado no solo insensibles a las necesidades del niño sino también rechazantes. En un principio, la conducta del niño parecerá saludable debido a su respuesta, aparentemente, independiente; sin embargo, son niños con dificultades emocionales (Oliva, 2004).

Además, Brando, Valera y Zárate (2008) plantean que, en este tipo de apego, existe incomodidad en las relaciones cuando hay cercanía emocional, por lo que 
se mantienen alejados, argumentando la importancia de ser independientes y autosuficientes. Para obtener aprobación social inhiben sentimientos negativos o agresivos; la información personal que brindan es muy limitada y, en su discurso no hay contacto con las emociones ya que no son valoradas al igual que el significado de las relaciones cercanas, por lo que el apego evitativo se define, principalmente por la inseguridad y la falta de confianza.

En el caso de los adultos, este tipo de apego se presenta en individuos que tuvieron y recuerdan una relación fría con su madre, un ambiente de crianza que generó sentimientos de rechazo y baja capacidad para responder a sus necesidades, por lo que en la adultez son personas que manifiestan reducida confianza personal y con los demás, con dificultades para mantener relaciones interpersonales y con altos niveles de distancia emocional (Gómez-Zapiain, Ortiz \& Gómez-Lope, 2012; Medina, Rivera \& Aguasvivas, 2016).

Apego inseguro ambivalente: los niños muestran una combinación de reacciones positivas y negativas hacia su madre. En una situación nueva, los niños están en contacto tan cercano con ella, que casi no exploran su ambiente, parecen ansiosos incluso antes que ella se separe y, cuando lo hace, muestran gran aflicción.

Cuando la madre regresa, muestran reacciones ambivalentes, tratan de estar cerca de ella pero también muestran gran enojo, dando golpes y pateando. Se ha determinado que alrededor de 10 a $15 \%$ de los niños tienen este tipo de apego.

A edades mayores, los niños con apego inseguro ambivalente muestran más dificultades en las relaciones, con comportamientos ambivalentes; por lo que son menos competentes en lo social y lo emocional, muestran conductas de irritación, resistencia al contacto y al acercamiento (Oliva, 2004).

Por su parte, las madres se comportan de manera incongruente; en ocasiones, se muestran muy atentas y en otras ignoran o rechazan los esfuerzos del niño por mantener el contacto; así lo contradictorio de su respuesta (Balbi, 2004) determina que el niño no sepa a qué atenerse, esto incrementa su ansiedad y se siente inseguro de la disponibilidad de su madre. Como estrategia para conseguir atención, el niño puede mostrar mucha dependencia y se ve impedido de desarrollar sus tareas evolutivas, todo esto como respuesta a la inestabilidad de la disponibilidad de sus figuras de apego (Oliva, 2004).

Para Brando, Valera y Zárate (2008) este tipo de apego caracteriza a individuos que anhelan la intimidad emocional pero se relacionan con personas que los rechazan de manera frecuente $\mathrm{y}$, como consecuencia, manifiestan conductas de apego exacerbadas lo que se explica con una historia vincular reiterada, en la que sus conductas de acercamiento se han visto frustradas, con frecuencia, por la baja o casi nula confianza en la figura de apego debido a su conducta inconstante de respuesta. Poseen gran necesidad de contacto e intimidad, pero a la vez temen perder el vínculo, lo que genera contradicción entre el deseo de cercanía y el temor a fracasar.

En el caso de los adultos, este tipo de apego, también denominado inseguro ansioso, es característico en personas que tuvieron y recuerdan una relación con una figura de apego poco comprensiva y ambivalente al momento de satisfacer sus necesidades, lo que genera individuos con altos niveles de desconfianza, inseguridad y sentimientos de abandono constante (Medina, Rivera \& Aguasvivas, 2016).

Aunque, en un principio, solo se propusieron estos tres estilos de apego, luego se describió un cuarto estilo, denominado apego inseguro desorganizado o desorientado (Oliva, 2004).

Apego desorganizado-desorientado: Los niños con este apego tienen varias conductas contradictorias y confusas que no encajan en ninguno de los otros estilos de apego; pueden correr hacia la madre cuando regresa pero no la miran. Al inicio del contacto, parecen tranquilizarse pero luego, de repente, rompen en un llanto de enojo. Su confusión sugiere que, tal vez, tengan el apego menos seguro de todos. Se ha determinado que un 5 a $10 \%$ de los niños se encuentran en esta situación.

Los niños y niñas con este tipo de apego, muestran más dificultades en las relaciones a edades mayores, 
con comportamientos confusos, por lo que son menos competentes en lo social y emocional; y ya como adultos, no se sienten cómodos abriéndose a los demás y dependiendo de ellos, por lo que evitan el contacto (Fraley, 2019).

La teoría del apego y sus tipos ha generado gran interés en la comunidad científica, incluso constituyen la base de nuevas propuestas como la de Barroso (2014) quien, usando un modelo de funcionamiento del sí mismo, propone cuatro tipos de apego adulto. (1) El 'estilo seguro' con una percepción positiva de sí mismo y de los otros, baja ansiedad y evitación ante contactos interpersonales. (2) El 'estilo desentendido o evitativo' tiene una idea positiva de sí mismo pero negativa de los otros; su ansiedad es baja pero la evitación alta. (3) Después viene el 'estilo preocupado' en el que la idea de sí mismo es negativa y la de los demás positiva; la ansiedad es alta y la evitación es baja. (4) Finalmente, el 'estilo temeroso' se caracteriza por una idea negativa tanto de sí mismo como de los otros, acompañada de ansiedad y evitación altas.

\section{TIPOS DE APEGO Y PROBLEMAS PSICOLÓGICOS Y RELACIONALES}

El apego es fundamental para el desarrollo social de la primera infancia y tiene efectos directos en el desenvolvimiento psicológico y relacional en la adolescencia y adultez. Como se pudo determinar en la descripción anterior, cada tipo de apego provoca la aparición de diferentes características que impactan sobremanera en el desempeño social de las personas.

En el caso de adultos, se entiende el apego como la tendencia estable que un ser humano posee para hacer grandes esfuerzos en la búsqueda y mantenimiento de la proximidad y el contacto con una o pocas personas específicas, que le proporcionan el potencial subjetivo para la seguridad física y psicológica. Esta tendencia se regula por modelos internos de trabajo, cognitivos, afectivos y motivacionales, que se construyen a partir de las experiencias del sujeto a nivel interpersonal (Sperling \& Berman, 1994).

Según lo planteado por Montebarocci, Codispoti, Baldaro \& Rossi (2004) los estudiosos del apego reconocen y describen la influencia que tienen las experiencias tempranas de los distintos tipos de apego en la representación evolutiva del 'yo' y los 'demás', siendo fundamental el denominado 'modelo de trabajo interno' del apego, que influye en las relaciones futuras y permite regular distintas estrategias en el adulto.

Diversas investigaciones han mostrado una clara relación entre bienestar o psicopatología en el adulto y el tipo de apego que poseen. Así, un estudio realizado con una muestra de adolescentes entre 13 y 17 años de edad, indicó que el apego ansioso evitativo está asociado, de manera directa y positiva, con sintomatología psicopatológica: ansiedad, obsesióncompulsión, depresión, hostilidad, entre otras (PintoCortez, Beyzaga, Cantero, Oviedo, \& Vergara, 2018).

También se ha demostrado que en la adultez, el apego inseguro tiene efectos sobre distintas sintomatologías depresivas. Una investigación realizada con un grupo de mujeres adultas entre 18 y 51 años, encontró que las pacientes que tenían un diagnóstico de algún tipo de depresión presentaban un estilo de apego inseguro y que las mujeres sin sintomatología depresiva presentaban un apego seguro (Garrido, Guzmán, Santelices, Vitriol, \& Baeza, 2015).

De igual forma, se ha determinado que ciertos trastornos, como el abuso de sustancias (Becoña, Fernández, Calafat, \& Fernández-Hermida, 2014) están relacionados con la conducta del padre más que con la de la madre, ya que, se sabe que los bebés pueden formar apegos hacia otros individuos diferentes.

Existe evidencia de que las experiencias negativas en las relaciones con los padres están vinculadas con ciertos problemas cognitivos (Bowlby, 1988): situaciones dolorosas que los padres no desean que los hijos conozcan (la muerte de alguien por suicidio) u otras como maltrato, abuso, violencia, etc., forman parte de estas categorías y pueden conducir a olvidos, amnesias, distorsiones e incluso formas de psicopatología más graves como alucinaciones o pensamientos paranoides, especialmente cuando existen amenazas de abandono o de rechazo de por medio.

De la misma manera, ciertas investigaciones realizadas con jóvenes y adultos universitarios 
coinciden en plantear que las personas con estilos más seguros de apego, presentan menos dificultades o confusiones emocionales, baja ansiedad y evitación, por lo que expresan de mejor manera sus emociones, tienden también a tener menores niveles de dependencia emocional y mayor estabilidad durante la etapa de noviazgo (Guzmán, Carrasco, Figueroa, Trabucco, \& Vilca, 2016; Valle \& La Moral, 2018), lo que sin duda repercute en el desarrollo de la relación intrapersonal e interpersonal.

Según lo destaca Balabanian, Lemos \& Vargas Rubilar (2015) siguiendo las ideas propuestas por Barudy \& Marquebreucq (2006), las diferentes investigaciones muestran que el apego de buena calidad, continuo y que se mantiene en el tiempo, es esencial para que las personas se desarrollen sanas, psicológica y emocionalmente, cuenten con herramientas que les permita afrontar distintas dificultades y se mantengan saludables durante la adolescencia y adultez.

Así mismo, la calidad de los diferentes estilos de apego que se construyen durante la infancia permiten predecir procesos relacionales y emocionales en la pubertad, adolescencia y adultez temprana, ya que se ha demostrado que el tipo y la calidad del apego influye en la capacidad de regulación emocional, sobre todo de aquellos adolescentes ambivalentes y evitativos que tienden a tener mayores desajustes emocionales (Oliva, 2011).

\section{INVESTIGACIONES SOBRE TIPOS DE APEGO}

Desde hace mucho tiempo atrás, existe interés por evaluar y valorar el tipo de apego relacionado con diferentes sintomatologías o aspectos positivos del desarrollo humano. Así, se encuentra que ciertas investigaciones (Flores, 2010) sugieren tres aspectos importantes del apego: (1) Es un elemento primordial en el desempeño afectivo y cognitivo de los seres humanos (Diamond \& Marrone, 2003); (2) el Sistema Nervioso Central de los mamíferos funciona por medio de bucles de retroalimentación abierta que requieren estabilización y regulación externa continua (Lewis, Amini, \& Lannon, 2000), la cual es aportada por las relaciones interpersonales (Schore, 2003) y (3) el requerimiento anterior es una necesidad biológica y no tiene edad ni etapa específica (Bowlby, 1998).
Otros trabajos (Flores, 2010) han definido tres funciones primarias del sistema de apego: (1) regulación de la proximidad del cuidador en un momento de estrés, (2) aporte de cuidado y seguridad (refugio) y (3) desarrollo de una base segura que permite la exploración del mundo alrededor que incluye, a largo plazo, el aprendizaje y el contacto con el conocimiento. Cierta evidencia más actual (Fonagy, Gergely, Jurist, \& Target, 2002) señala otra función respecto a la posibilidad de mentalización facilitada por la relación con los otros (Grande-García, 2009).

De igual manera, se ha determinado que existe una fuerte relación entre los vínculos emocionales y los 'sistemas de acción' (estructuras implicadas en el procesamiento de la información) (Panksepp, 1998); el más básico es la búsqueda de una relación para obtener cuidados.

Este sistema de acción se caracteriza por estar entrelazado con circuitos neurobiológicos, determinados genéticamente, implicados en las reacciones emocionales y su regulación y responden de forma incondicionada a los estímulos, produciendo secuencias conductuales organizadas (Schore, 1994). Lo cual comprueba la importancia de los vínculos para el control y gestión de los aspectos emocionales.

El esquema interpersonal se graba en los niveles inferiores del cerebro y se mantiene como una huella somatosensorial que satura y condiciona automáticamente, la forma de relacionarse, sin que la parte consciente tenga acceso. Este aspecto también ha sido evidenciado por la investigación de Soon, Braas y Haynes (2008) que muestra la reacción de una persona, milisegundos antes de que la conciencia se dé cuenta de lo que está sucediendo; esto comprueba que la realidad inconsciente y visceral reacciona antes $\mathrm{y}$, posteriormente, se da un procesamiento cognitivo más avanzado que solo se puede lograr cuando la información llega al neocórtex (Salvador, 2013).

Así, a través de la evolución, el cerebro se convirtió en un órgano muy especializado y sofisticado que integra la experiencia humana (interna y externa), siempre y cuando, las situaciones sean adecuadas para el nivel de desarrollo propio de cada edad, con diferentes capacidades y niveles de maduración. 
De igual manera se ha encontrado que el funcionamiento del sistema nervioso está afectado por la presencia de hormonas tales como la oxitocina, la vasopresina y las endorfinas que tienen participación en el establecimiento del vínculo entre madre e hijo (Olza, 2008).

Si el ambiente es estable y seguro, la experiencia también lo es y no se activa ninguna respuesta de estrés frente a una situación que el cerebro límbico puede considerar amenazante. Por el contrario, si el ambiente es inestable e inseguro, de igual manera, la experiencia también es así y, la respuesta emocional, se activa automáticamente como si existiera un peligro para la supervivencia, situaciones que pueden tener gran impacto en el ámbito del desempeño a todo nivel, ya que si el sujeto percibe una situación como amenazante, los circuitos de respuesta se activan automáticamente.

Siguiendo a Flores (2010), se afirma entonces que: "los seres humanos regulan los unos a los otros, su fisiología y modifican la estructura interna del sistema nervioso de los otros a través del intercambio sincrónico de emociones. Esta es la base para el apego y la memoria de esto se graba en el cambio que se experimenta" (p. 551).

De esta manera, la investigación del apego es un área muy interesante de desarrollar, puesto que se ha relacionado con ciertas características neuropsicológicas y, en consecuencia, tiene impacto sobre sus funciones. Así, el apego seguro se ha vinculado con la maduración fisiológica del cerebro (Cozolino, 2006; Siegel, 1999), la capacidad de regulación afectiva (Fonagy, Gergely, Jurist, \& Target, 2002), las representaciones internalizadas que organizan e influyen en la conducta hacia los otros (Diamond \& Marrone, 2003), las capacidades cognitivas, la autoestima (Mikulincer \& Shaver, 2007) y la autoimagen (Lewis, Amini, \& Lannon, 2000).

De igual manera, se ha desarrollado una línea de investigación estudiando las relaciones amorosas para encontrar paralelos entre las cualidades de apegos infantiles con las relaciones de pareja en la adultez (Martínez y Santelices, 2005).
Recientes estudios realizados en Canadá, han investigado los diferentes estilos de apego en sobrevivientes de abuso sexual en la infancia, en donde se asegura que en la adultez pueden desarrollar un estilo de apego elevado en ansiedad o un apego evitativo, presentando además una compulsión sexual o una evitación de contacto sexual (Labadie, Vaillancourt-Morel, Godbout, N. \& Sabourin, 2018). El estudio concluye que las personas que han sido abusadas sexualmente durante su infancia, presentan fuertes conflictos en el desarrollo del apego, por lo que existen repercusiones psicológicas que permanecen muchos años después del trauma.

Una revisión confirmó que existe una relación negativa entre el apego inseguro y la satisfacción de la relación, en donde el efecto es más fuerte en el miembro de la pareja evaluado que en su pareja, aunque también depende de otros factores como la edad, el estado y tiempo de la relación entre otros (Candel \& Turliuc, 2019). En el estudio surgieron 15 perfiles diferentes, entre los cuales destacan dos: (1) mujeres que presentan gran ansiedad, con dificultades en su sexualidad y, (2) varones con apego inseguro y problemas de ambivalencia en su sexualidad.

En cuanto a estudios reportados entre la relación de los estilos de apego y algunos trastornos psicológicos, se mencionan algunos de ellos a continuación.

Una investigación buscó la correspondencia entre el maltrato parental en la infancia, el estilo de apego y su influencia en los síntomas relacionados con la personalidad límite. Se encontró que en las mujeres, el maltrato de sus padres y en hombres el maltrato de sus madres, está directamente asociado con los síntomas de personalidad límite desde el estilo de apego ansioso (inseguro) pero no el evitativo (Godbut, Daspe, Runts, \& Cyr, 2019).

La relación entre el apego inseguro y niños con déficit de atención e hiperactividad también ha sido estudiada, aunque los resultados aún no son concluyentes (Rasmussen, Bilenberg, Shmueli-Goetz, Simonsen, \& Bo Bojesen, 2019).

Los estilos de apego incluso han sido estudiados en relación con la adicción al internet, encontrándose 
una relación significativamente positiva entre el estilo de apego inseguro (ansioso y evitativo) y un uso más intensivo y disfuncional de redes sociales, como una manera de reemplazar y compensar los afectos no recibidos del medio cercano del individuo (D'Arienzo, Boursier, \& Griffiths, 2019).

\section{MÉTODOS PARA EVALUAR EL APEGO}

Las primeras propuestas metodológicas para valorar el apego fueron realizados de forma experimental, mediante lo que Ainsworth (Chamorro, 2012) denominó la situación del extraño, en donde se ponían en evidencia los tres tipos de apego, a través de la exposición de niños a tres situaciones: (a) acompañado con la presencia de su cuidador, (b) acompañado de su cuidador y la presencia de un extraño y (c) solamente con la presencia de extraño; permitiendo así valorar el tipo de apego que presentaba el niño.

A partir de este avance en la operacionalización de los fundamentos teóricos del apego, se han propuesto diversos instrumentos para valorarlo, resaltando la construcción de diversas escalas, por lo que se hace una revisión de algunas de ellas a continuación.

En 1985, George, Kaplan y Main diseñan el cuestionario 'Adult Attachment Inventory (AAI)' para evaluar el modelo interno activo en adultos (Oliva, 2004). El objetivo de este cuestionario es preguntar al sujeto sus recuerdos sobre sus experiencias de apego en la infancia y la valoración que le han dado. Lo importante es la interpretación que se ha hecho de estas experiencias mas no la experiencia en sí.

A partir de esta evaluación se encuentran tres tipos de modelos internos activos. El primero es de padres seguros o autónomos que muestran, tanto coherencia como equilibrio, al valorar las experiencias infantiles. Luego, están los padres preocupados, quienes muestran emoción al recordar experiencias de la infancia, en especial ira hacia sus padres; la relación con sus padres les causa confusión y no pueden ofrecer una imagen clara o sin contradicciones.

Finalmente, están los padres rechazados, quienes no le dan importancia a sus vivencias infantiles de apego, idealizan a los padres sin recordar concretamente alguna experiencia; sus expresiones no revelan emoción, son intelectuales y frías. Luego, se añade una cuarta categoría que son los padres no resueltos, quienes equivaldrían al apego inseguro desorganizado.

También se reporta la Escala de Desarrollo Emocional (EDS por sus siglas en inglés) (Hart \& Jacobsen, 2019), la cual ha sido desarrollada en el marco teórico de la Psicología del Desarrollo Neuroafectivo (NADP), estrechamente relacionado con el constructo de apego. Esta escala se presenta como un apoyo para la comprensión de los patrones esperados de desarrollo emocional y su desenvolvimiento a lo largo de la vida.

Su relevancia está dada ya que después de realizar una revisión sistemática de la literatura (Hart S., 2018), encuentra que muchos métodos de evaluación están basados en observaciones que tan sólo abordan el comportamiento y los síntomas de los niños mas no su desarrollo emocional.

El estudio se realizó con 36 familias de 248 niños entre 4 a 12 años de edad, de los cuales 64 habían sido referidos por la institución educativa o instituciones psiquiátricas y 148 no habían sido referidos por ninguna institución. Se aplicó la escala que busca la correlación entre procesos neuropsicológicos con el desarrollo emocional, principalmente los sistemas de autonomía, funcionamiento límbico y prefrontal.

Este instrumento consta de dos sub-escalas (EDS-P/EDS-A) diseñadas para evaluar el grado de funcionamiento emocional actual a nivel autonómico, límbico y prefrontal en los niños, de diversos centros de tratamiento familiar en Dinamarca (Hart \& Jacobsen, 2019). Las propiedades psicométricas del estudio piloto incluyeron análisis de confiabilidad entre evaluadores (.918-.986; $p<0.05)$, confiabilidad test-retest con una correlación positiva significativa (.805-.930; p < 0.05) y buena consistencia interna (Alfa de Cronbach $\alpha=.813$ ).

Sin embargo, las correlaciones entre las puntuaciones oscilaron entre $\mathrm{r}=.084 \mathrm{y} \mathrm{r}=.400$. Además, la validación del constructo fue analizada al correlacionar los resultados de la EDS entre grupos 
referidos y no referidos a servicios regionales de asesoramiento psicopedagógico y psiquiátrico infantil, existiendo mayor autonomía y control de impulsos en el segundo grupo.

A partir de los resultados del estudio piloto, se concluye que esta escala parece ser una herramienta confiable y parcialmente válida que podría ayudar a evaluar el desarrollo y competencias emocionales, ya que se encontró que existen diferencias considerables y significativas entre un niño evaluado dentro de un contexto controlado a uno que se encuentre en su entorno natural.

Esto confirma la teoría expuesta, puesto que un ambiente tranquilo y seguro genera mejores estados neurofisiológicos y contribuyen a una mejor regulación emocional, lo cual también se encontró en la investigación, ya que se hallaron mayores progresos emocionales en niños que viven en un ambiente estable y seguro brindado por sus cuidadores (Hart \& Lindahl, 2019). Sin embargo, se plantea la necesidad de mayor investigación sobre sus propiedades psicométricas y sus aplicaciones clínicas.

Otro instrumento propuesto es el Cuestionario de Apego y Problemas Clínicos (ACIQ, por sus siglas en inglés) (Lindberg \& Thomas, 2011); se trata de una batería que contiene 29 escalas; mide el apego (a padre, madre, pareja) y problemas clínicos relacionados. Fue planteado con respuestas de cuatro puntos tipo Likert.

La correlación de cada ítem con su respectiva escala y su relación con la escala global muestra un Alpha de Cronbach de .79. El análisis factorial demuestra que las escalas se apoyan en figuras de apego y problemas clínicos en lugar de los estilos de apego, en contraste con los supuestos de la teoría tradicional.

Un aporte significativo está dado por el estudio de Lindberg y Zeid (2018) con una muestra de 206 presos y 239 reclusas, cuyos resultados arrojaron un Alpha de Cronbach de .80. Esta investigación parte de la Teoría de Apego y de los Sistemas Dinámicos del Desarrollo del Crimen (ADDSTOC) considerando además, la psicopatología del desarrollo lo que permite enfocarse en las diferencias individuales de los patrones causales criminales; es decir, integra las influencias biológicas, psicológicas y sociales (apego inseguro, eventos adversos en la infancia, adicción al crimen y relacionamiento con compañeros de crimen) en un modelo significativo para predicción del comportamiento criminal.

Aunque la literatura existente ha abordado cada uno de estos factores de manera aislada, Lindberg y Zeid (2018) argumentan que la validación empírica de un sistema complejo e interactivo es esencial para la conceptualización adecuada del desarrollo de la criminalidad (incluyendo un estudio y tratamiento personalizados), ya que cada uno de estos componentes depende de los demás en su poder predictivo.

Por otro lado, se tiene estudios como el de Gander y colegas (2018) en el que describen el uso del "Sistema de Imagen Proyectiva de Apego en el Adulto" (AAP, por sus siglas en inglés), en el caso de un adolescente iraquí refugiado en Austria y con diagnóstico de trastorno de estrés post-traumático (PTSD). Este sistema fue diseñado para evaluar el estado de apego, consiste en un conjunto de estímulos de imágenes que incluyen un dibujo neutral y siete dibujos que representan escenas como separación, enfermedad, soledad, muerte y abuso.

Las pruebas realizadas en cuanto se refiere a las propiedades psicométricas evidencian una confiabilidad entre evaluadores del $90 \%, x=.85, \mathrm{p}<$ .001 ; test-retest de .84 (a lo largo de tres meses); una validez concurrente con la Entrevista de Apego en el Adulto (AAI) (la tasa de concordancia para los cuatro grupos fue de $90 \%, x=.84, \mathrm{p}<.001$ y para los dos grupos [seguros e inseguros] fue de $97 \%, x=.89, \mathrm{p}$ $<.001$ ); y, una validez discriminante entre pacientes clínicos y grupos de control saludables (Gander, Diamond, Buchheim, \& Sevecke, 2018).

$\mathrm{Su}$ evaluación multifacética a través de otras pruebas (AAP; Entrevista Clínica Estructurada eje I y II; Escala Wechsler de Inteligencia y el Youth SelfReport) sirvió para mostrar la manera en que una medida narrativa, validada y confiable para evaluar las representaciones de apego, se puede utilizar para ampliar la comprensión de la psicopatología del individuo, es decir, profundizar más allá de los 
patrones de apego, hacia un análisis de los patrones de representación y defensa en la narrativa.

Este análisis proporciona, sin duda alguna, una visión más profunda del funcionamiento, tanto al interior como más allá del sistema familiar. Además, aporta una descripción general de la forma en que las intervenciones terapéuticas podrían centrarse en ayudar a los pacientes a comprender sus reacciones emocionales de impotencia, en el contexto de su tratamiento.

De igual manera, también existe una versión adaptada y validada en idioma polaco (Adamczyk, et al., 2019) de la Escala de Miedo a estar Soltero (FBSS, por sus siglas en inglés) (Spielmann, et al., 2013), la cual busca confirmar y profundizar los hallazgos del estudio original en un contexto polaco.

Dicho constructo y escala se han desarrollado en referencia a otras medidas de inseguridad, como el apego ansioso, y su énfasis está en que el miedo a estar soltero puede demostrar la especificidad de la figura de apego deseada por los individuos, mientras que el apego ansioso puede centrarse en las figuras de apego más allá de las parejas meramente románticas.

Se trata de una escala de seis ítems diseñada para medir el miedo de estar soltero; las respuestas se valoran entre 1 (no del todo cierto) a 5 (muy cierto). En una serie de estudios, la consistencia interna de la escala original varió desde $\alpha=.83$ a $\alpha=.87$ (Spielmann, et al., 2013). En el estudio mencionado, participaron 175 personas $(60 \%$ mujeres, $40 \%$ hombres, tanto solteros y en pareja) de entre 18 y 67 años. Se emplearon ocho formas de medición entre escalas y cuestionarios de los cuales, dos serían fundamentales tanto para el estudio de Adamczyk y colegas como para el presente estudio.

Por otro lado, también se conoce la Adult Attachment Scale (AAS) (Collins, 1996) que consta de 18 ítems que miden tres dimensiones de apego (aproximación, evitación, ansiedad) y los cuatro tipos de apego (seguro, rechazante, preocupado, temeroso). En el estudio de Adamczyk y colegas (2019), las alphas de Cronbach se habrían determinado $\alpha=.77, \alpha$ $=.71 \mathrm{y} \alpha=.88$ para las subescalas de Aproximación, Evitación y Ansiedad, respectivamente.
$\mathrm{El}$ análisis factorial exploratorio provee evidencia de que el miedo a estar soltero y el apego representan construcciones distintas; sin embargo, el ítem \# 1 del FBSS no carga en el factor \# 2 ('Miedo a estar soltero') y tampoco en el factor \# 1 ('Apego ansioso). A través de la validez de constructo se demuestra que los dos grupos (solteros y parejas) no difirieron en cuanto al miedo a estar solteros.

Se evaluó la validez convergente y discriminante del FBSS, encontrándose que el miedo a estar soltero se relaciona positivamente con el apego ansioso y este predijo el miedo a estar soltero. Por último, a través de la Alpha de Cronbach se estableció que la confiabilidad del FBSS polaco es alta.

Además, al ampliar el estudio original de Spielmann y sus colegas (2013), la investigación de Adamczyk y colegas (2019) también determinó una confiabilidad test-retest del FBSS polaco de $r$ $=.77$ durante un período de seis meses y este valor representa una fuerte correlación.

Michael y Snow (2019) revisaron las propiedades psicométricas de la Escala para Adultos de Apego Parental - Forma Reducida (ASPA-SF, por sus siglas en inglés) con el objetivo de obtener información sobre la memoria perceptiva de un adulto respecto a los patrones de relación con las figuras de apego que tuvo en la infancia. El estudio se ha reportado en la Universidad Tecnológica de Tennessee y se realizó en base a una muestra de 250 encuestados.

La puntuación media de las participantes es $\mathrm{M}=$ 37.52; el apego seguro para la madre superó el de los participantes masculinos $(\mathrm{M}=34.31), \mathrm{t}(545)=4.05$, $\mathrm{p}<.001$. Además, los investigadores declararon que las participantes femeninas $(\mathrm{M}=10.61)$ obtuvieron calificaciones más altas que los hombres $(\mathrm{M}=9.68)$ en relación con la madre, $\mathrm{t}(556)=2.62, \mathrm{p}=.009$. dependiente de la madre. Se hizo referencia a la puntuación media de las participantes femeninas $(\mathrm{M}=$ 12.70) como superior a la de los hombres $(M=11.66)$, $\mathrm{t}(553)=2.84, \mathrm{p}=.005$.

Estos aspectos permiten concluir que los resultados proporcionan información adecuada sobre las propiedades psicométricas de la escala; sin embargo, 
existe la necesidad de nuevas investigaciones que impliquen el uso de la ASPA-SF, ya que las variables a evaluar fueron muy generales y pueden existir más temáticas de estudio en base a los patrones de relación.

Sin embargo, este estudio ha proporcionado las pruebas suficientes de que la ASPA-SF tiene la capacidad de ser un instrumento con valor para el desarrollo y la comprobación de las teorías, ya que ofrece la oportunidad de proporcionar a los asesores y clientes, información sustentable respecto a los patrones de relación que existe con los cuidadores de la infancia.

Este estudio es de gran aporte para la presente investigación, debido a que, a pesar de las variables que se pudieron añadir en la escala, engloba gran parte de lo que interesa, es decir proporciona apoyo a la utilización de la ASPA-SF en contextos de orientación con los estudiantes de grado y posgrado. De esta manera, el estudio muestra el proceso de creación de una versión abreviada a través de análisis factorial exploratorio y un análisis factorial confirmatorio, haciendo un instrumento válido para evaluar la teoría del apego.

Otra investigación (Darling Rasmussen, P.; Bilenberg, N.; Shmueli-Goetz, Y.; Simonsen, E.; Bo Bojesen, A.; Storeb, O. 2019) acerca de las Representaciones de Apego en madres con hijos diagnosticados con TDAH, tuvo como objetivo investigar la distribución de la transmisión intergeneracional y el impacto potencial de las representaciones de apego infantil en respuesta al tratamiento en una muestra de niños con TDAH, en la región de Zelanda-Dinamarca.

Esta investigación se propuso debido a que se sabe muy poco acerca de la distribución y transmisión de la representaciones de apego en poblaciones de niños que padecen TDAH, lo que permitiría a largo plazo, evaluar la influencia que existe entre el pronóstico de TDAH y el apego.

En cuestión de la metodología, se reclutaron sesenta personas, madre-hijo con pronóstico de TDAH. Se entrevistó a los padres para evaluar el apego adulto (AAI) y la entrevista de Apego Infantil (CAI). la severidad de los síntomas del TDAH se evaluó en cuatro puntos con la Escala de Calificación del TDAH. Los resultados indicaron una notable prevalencia de apego inseguro, tanto en los niños como en las madres.

El estudio concluye que el apego inseguro depende de muchos factores; sin embargo, permanecen como variables importantes a considerar, la importancia de la fijación como un factor en el tratamiento, el deterioro funcional y el pronóstico a largo plazo de los niños con TDAH, lo cual, subraya la importancia de entender ciertas variables que determinan un apego seguro o inseguro y su relación con algunos trastornos infantiles.

En Portugal, Corval, Belsky, Baptista, Mesquita y Soares (2019) llevaron a cabo una investigación para desarrollar y validar, con una medida observacional de síntomas, el trastorno de apego reactivo. Esta herramienta se usa con niños en edad preescolar, en los cuales también se mide el apego reactivo, con 17 calificaciones, agrupados en tres subescalas que evalúan el apego, comportamiento exploratorio y socioemocional,

En la muestra participaron 134 preescolares institucionalizados, con una media de edad de $\mathrm{M}=54.84$ meses $(\mathrm{SD}=10.83 ; 60 \%$ niños $) \mathrm{y}$ sus cuidadores. Se encontró una confiabilidad adecuada para las subescalas. Con respecto a la calidad de la relación cuidador-niño, las puntuaciones totales de esta escala, se asociaron de forma moderada e inversa con la presencia de una relación selectiva entre el niño y el cuidador, como se esperaba; de hecho, también surgió una asociación significativa y negativa para cada una de las tres subescalas.

El instrumento resultó ser confiable y válido, aunque no todas las hipótesis fueron confirmadas ya que la muestra estaba compuesta por niños preescolares portugueses institucionalizados que ya tenían una historia de rasgos patológicos, lo que limita la generalización de los hallazgos. La idea sería realizar la misma investigación con esta escala pero con diferentes muestras.

Lee y Shin (2019) llevaron a cabo la validación de la Escala de Experiencia en Relaciones Cercanas 
en versión reducida (ECR-S por sus siglas en inglés), con una muestra de estudiantes coreanos. El instrumento confirma que los estilos de apego se midieron originalmente como variables categóricas, identificando tres estilos de apego romántico: seguro, ansioso-ambivalente y evitativo.

Participaron 229 (60.7\%) hombres y 148 (39.3\%) mujeres, de 19 a 34 años, con una media de edad de 22.92. Los niveles de educación fueron 84 (22.3\%) estudiantes de primer año, 127 (33.7\%) estudiantes de segundo año, 92 (24.4\%) juniors, y 73 (19.3\%) seniors; 156 (41.4\%) personas estaban actualmente en relaciones y $219(58.1 \%)$ eran solteros y estaban sin pareja. Los estudiantes coreanos fueron reclutados en dos grandes universidades ubicadas en Seúl, Corea del Sur.

Los instrumentos fueron traducidos al coreano con una revisión lingüística por parte de un experto bilingue, excepto la Escala de soledad de la UCLA y el resumen. La versión traducida de las encuestas se comparó con la original y se volvió a traducir, cuando se encontró un error en el significado hasta alcanzar una congruencia adecuada. La versión coreana de la encuesta fue entregada y completada por los participantes que aceptaron participar en el estudio.

Como se esperaba, la búsqueda excesiva de tranquilidad está asociada significativamente y de forma positiva con la ansiedad $(\mathrm{r}=.34)$ pero no con la evitación $(\mathrm{r}=-.01)$. La búsqueda de apoyo se asoció significativa y positivamente con la ansiedad $(r=.21)$ pero significativa y negativamente con la evitación ( $\mathrm{r}$ $=-.57)$.

Adicionalmente, la depresión se asoció de manera significativa y positiva con la ansiedad $(\mathrm{r}=.39)$ y evitación $(\mathrm{r}=.14)$, mientras que la satisfacción de la relación se asoció significativa y negativamente tanto con la ansiedad como la evitación $(\mathrm{r}=-.12)$. En conjunto, estos hallazgos brindan un apoyo considerable para la validez concurrente y discriminante de las puntuaciones coreanas de ECR-S.

Además, se observó una clara discriminación de apego por una débil correlación entre la subescala de ansiedad y evitación $(\mathrm{r}=.09)$. Las consistencias internas para las subescalas ECR-S fueron .81 por ansiedad y .70 por evitación. Así, el ECR-S coreano mostró una consistencia interna similar.

$\mathrm{Su}$ aporte es interesante para la presente investigación respecto a los resultados que concluyen que existen diferencias de apego individual que incluyen la ansiedad y evitación. Las personas con alta ansiedad demuestran una gran necesidad de cercanía, exigiendo la atención de su pareja y exhibiendo un comportamiento de aferramiento. Estos individuos exhiben una estrategia de gestión del efecto hiperactivo, que requiere la atención constante de su pareja. Por el contrario, el apego evitativo se caracteriza por una desactivación de la gestión del afecto, incluida su regulación, al distanciarse del compañero, al tiempo que se suprimen las señales relacionadas con el apego. Las personas con un alto apego evitativo muestran incomodidad con la cercanía a personas pero son autosuficientes al realizar tareas.

Otro estudio (Cadman, Belsky \& Pasco Fearon, 2018) propuso el desarrollo de la Escala breve de Apego (BAS-16 por sus siglas en inglés) basándose en el cuestionario Q-Sort (AQS), una de las medidas estándar apego. Este estudio se llevó a cabo en Londres, en el Reino Unido.

El BAS-16 se creó con dos escalas de ocho elementos, relacionadas con la interacción armoniosa con el cuidador y las conductas de búsqueda de proximidad; mostró una validez convergente, discriminante y concurrente comparable al AQS completo. Algunas de sus propiedades psicométricas indican que la subescala de interacción armoniosa y la puntuación total de BAS-16 se correlacionan significativamente con la sensibilidad, la externalización y la competencia social, a una magnitud similar a la AQS completa; por el contrario, la escala de búsqueda de proximidad mostró sólo una débil correlación con externalización, ni el BAS-16 ni el AQS completo tuvieron una correlación significativa con el temperamento (valores $r$ entre -.07 y .06)

Gracias a este estudio y partiendo del análisis del factor de calidad, se han encontrado cuatro factores asociados con la relación, como son las características de interacción armoniosa, la búsqueda de proximidad, 
el retraimiento y la falta de coherencia. Estos factores permiten establecer correlaciones entre los distintos ítems de la escala abreviada. Se concluye que el apego seguro se encuentra, principalmente, en los niños que mantienen una relación armoniosa con sus padres y el apego desorganizado se encuentra ligado a conductas de incumplimiento e inquietud.

El estudio concluye señalando que la escala abreviada funciona de manera similar a la escala completa de apego y es efectiva para detectar apego inseguro de manera temprana. Sin embargo, no cubre todos los componentes de la escala completa (Cadman, Belsky, \& Pasco, 2018), por lo que muestra el potencial como una medida de detección para el apego inseguro en la infancia y, por lo tanto, señala una línea promisoria de investigación, al dejar abierta la posibilidad de validación de los otros segmentos de la escala.

De la sistematización realizada se puede llegar a algunas conclusiones: (a) la valoración del apego todavía es parte de una línea de investigación que se encuentra en desarrollo, (b) existe una carencia de escalas para valorar el apego en adultos, que hayan sido desarrolladas y validadas en América Latina, (c) muchas de las propuestas para valorar el apego se basan en el déficit o desde su valoración en negativo, (d) no se tiene un libre acceso a su contenido desde contextos latinoamericanos, (e) existe poca investigación que estudie los tipos de apego en estudiantes universitarios e, (f) insuficiente evidencia que demuestre buenas propiedades psicométricas de las escalas que se están utilizando con estudiantes universitarios.

\section{REFERENCIAS}

Adamczyk, K., Trepanowski, R., Celejewska, A., Kosińska, J., Mamot, A., Palczewska, M. (2019). The Polish adaptation and further validation of the Fear of Being Single Scale (FBSS). Current Psychology, 1-11. https://doi.org/10.1007/ s12144-019-00192-2.

Armus, M., Duhalde, C., Oliver, M. y Wosxobroinik, N. (2012). Desarrollo emocional. Clave para la primera infancia. Buenos Aires: UNICEF.
Balabanian, C., Lemos, V. y Vargas Rubilar, J. (2015). Apego percibido y conducta prosocial en adolescentes. Revista Colombiana de Ciencias Sociales, 6(2), 278-294.

Balbi, J. (2004). La mente narrativa. Hacia una concepción post-racionalista de la identidad personal. Buenos Aires: Paidós.

Barudy, J., \& Marquebreucq, A. P. (2006). Hijas e hijos de madres resilientes: Traumas infantiles en situaciones extremas: violencia de género, guerra, genocidio, persecución y exilio. España: Gedisa.

Barroso, O. (2014). El apego adulto: la relación de los estilos de apego desarrollados en la infancia en la elección y las dinámicas de pareja. Revista Digital de Medicina Psicosomática y Psicoterapia, 4(1), 1-25. Retrieved from http://www.psicociencias. com/pdf_noticias/Apego_Adulto.pdf

Becoña, E., Fernández, E., Calafat, A., FernándezHermida,J. (2014) Apego y consumo de sustancias en la adolescencia. Una revisión de aspectos conceptuales y metodológicos. Adicciones, 26(1), 77-86.

Bowlby, J. (1988). El papel de la experiencia de la infancia en el trastorno cognitivo. En M. Mahoney y A. Freeman. Cognición y Psicoterapia. p. 203222.

Bowlby, J. (1998). El apego. Barcelona: Paidós.

Brando, M., Valera, J. M., \& Zarate, Y. (2008). Estilos de apego y agresividad en adolescentes. Psicología-Segunda Época, 27(1), 16-42.

Cadman, T., Belsky, J., \& Pasco Fearon, R. M. (2018). The Brief Attachment Scale (BAS-16): A short measure of infant attachment. Child Care Health Dev. 44, 766-775, doi: 10.1111/2Fcch.12599.

Candel, O. S., \& Turliuc, M. N. (2019). Insecure attachment and relationship satisfaction: A meta-analysis of actor and partner associations. Personality and Individual Differences, 190-199 doi: 10.1016/j.paid.2019.04.037.

Casullo, M., \& Fernández Liporace, M. (2005). Evaluación de los estilos de apego en adultos. Anuario de investigaciones, 12, 183-192. 
Chamorro, L. (2012). El apego. Su importancia para el pediatra. Pediatria (Asunción), 9(3), 199-206.

Collins, N. (1996). Working models of attachment: Implications for explanation, emotion, and behavior. Journal of Personality and Social Psychology, 71(4), 810-832. https://doi. org/10.1037/0022- 3514.71.4.810.

Corval, R., Belsky, J., Baptista, J., Mesquita, A., \& Soares, I. (2019). Development and validation of an observational measure of symptoms of Reactive Attachment Disorder. Attachment \& Human Development, 21(2), p. 111-131, doi: 10.1080/14616734.2018.1499209.

Cozolino, L. (2006). The Neuroscience of Human relationships: Attachment and Developing Brain. New York: Norton.

Darling Rasmussen, P., Bilenberg, N., Shmueli Goetz, Y., Simonsen, E., Bo Bojesen, A., \& Storeb $\varnothing$, O. (2019). Attachment Representations in Mothers and Their Children, 28. Journal of Child and Family Studies, p. 1018-1028. doi: 10.1007/ s10826-019-01344-5.

D'Arienzo, M. C., Boursier, V., \& Griffiths, M. D. (2019). Addiction to Social Media and Attachment Styles: A systematic Literature Review. International Journal of Mental Health and Addiction., doi: 10.1007/s11469-019-000825.

Diamond, N., Marrone, M. (2003). Attachment and Intersubjectivity. London: Whurr.

Flores, P. (2010). Group Psychotherapy and Neuroplasticity: An Attachment Theory Perspective. International Journal of Group Psychotherapy, 60(4), p. 546-570.

Fonagy, P. Gergerly, G. Jurist, E., Target, M. (2002). Affect Regulation, Mentalization and the Development of the self. New York: Other Press.

Fraley, R. C. (2019). Attachment in adulthood: Recent developments, emerging debates, and future directions. Annual Review of Psychology, 70, 401422.

Gander, M., Diamond, D., Buchheim, A., \& Sevecke, K. (2018). Use of the Adult Attachment Projective Picture System in the formulation of a case of an adolescent refugee with PTSD. Journal of Trauma \& Dissociation, 19(5), p. 572-595. doi: 10.1080/15299732.2018.1451803.

Grande-García, I. (2009). Neurociencia social: el maridaje entre la Psicología Social y las Neurociencias Cognitivas. Revisión e introducción a una nueva disciplina. Anales de Psicología, 25(1), 1-20.

Garrido, L., Guzmán, M., Santelices, M. P., Vitriol, V., \& Baeza, E. (2015). Estudio comparativo de los estilos de apego adulto en un grupo de mujeres con y sin diagnóstico de depresión. Terapia psicológica, 33(3), 285-295. https://doi. org/10.4067/S0718-48082015000300011

Godbut, N., Daspe, M. E., Runts, M., \& Cyr, G. (2019). Childhood Maltreatment, Attachment, and Borderline Personality - Related symptoms: gender-specific structural equation model. Psychological Trauma: Theory, Research, Practice and Policy, 11(1), 90-98. doi:10.1037/ tra0000403.

Gómez-Zapiain, J., Ortiz, M.J., y Gómez Lope, J. (2012). Capacidad para aportar y solicitar apoyo emocional en las relaciones de pareja en relación con los padre de apego. Anales de Psicología, 28, 302-312

Guzmán, M., Carrasco, N., Figueroa, P., Trabucco, C., \& Vilca,D. (2016). Estilos de Apego y dificultades de Regulación Emocional en estudiantes universitarios. Psykhe (Santiago), 25(1), 1-13. https://doi.org/10.7764/psykhe.25.1.706

Hart, S. (2018). Psychometric properties of the emotional development scale: Investigating reliability and validity including correlating with marschakinteraction method and theneuroaffective mentalizing interview. Aalborg University. Aalborg, Denmark: Retrieved from: https://www. mt-phd.aau.dk/digitalAssets/435/435712_phd_ thesis_susan_hart.pdf

Hart, S., \& Jacobsen, S. L. (2019). The Emotional Development Scale: Assessing the Emotional Capacity of 4-12 Year Olds. Journal of Infant, Child, and Adolescent Psychotherapy, doi: 10.1080/15289168.2019.1583056 . 
Labadie, C., Vaillancourt-Morel, M., Godbout, N. \& Sabourin, S. (2018). Adults profiles of child sexual abuse survivors: attachment insecurity, sexual compulsivity, and sexual avoidance. Journal of Sex \& Marital Therapy, 44(4), 354-369. doi: 10.1080/0092623X.217.1405302.

Lee, J.-Y., \& Shin, Y.-J. (2019). Experience in Close Relationships Scale-Short Version (ECR-S) Validation with Korean College Students. Measurement and Evaluation in Counseling and Development, 52(2), 119-127, doi: $10.1080 / 07481756.2018 .1497431$.

Lewis, T., Amini, F., Lannon, R. (2000). A General Theory of Love. New York: Random House.

Lindberg, M., \& Thomas, S. (2011). The Attachment and Clinical Issues Questionnaire (ACIQ): Scale Development. The Journal of Genetic Psychology, 172(4), 329 -352. http://dx.doi.org/10.1080/0022 1325.2010.541382.

Lindberg,M.,\&Zeid,D.(2018). Tests of the Attachment and Developmental Dynamic Systems Theory of Crime (ADDSTOC): Toward a Differential RDoC Diagnostic and Treatment Approach. International Journal of Offender Therapy and Comparative Criminology, 62(12), 3746-3774. https://doi. org/10.1177/0306624X17750353.

Martínez, C., \& Santelices, M. P. (2005). Evaluación del Apego en el Adulto: Una Revisión. Psykhe, 14(1), 181-191. https://dx.doi.org/10.4067/ S0718-22282005000100014.

Medina, C. J., Rivera, L. Y., \& Aguasvivas, J. A. (2016). El apego adulto y la calidad percibida de las relaciones de pareja: Evidencias a partir de una población adulta joven. Salud \& Sociedad, 7(3), 306-318.

Michael, T., \& Snow, M. (2019). The Adult Scale of Parental Attachment-Short Form: Psychometric Properties, Factor Analyses, and Validation. International Journal for the Advancement of Counselling, 29. doi:10.1007/s10447-019-093759.

Mikulincer, M., Shaver, P. (2007). Attachment in Adulthood: Structure, Dynamics and Change. New York: Guilford.
Montebarocci, O., Codispoti, M., Baldaro, B., \& Rossi, N. (2004). Adult attachment style and alexithymia. Personality and Individual Differences, 36(3), 499-507. https://doi.org/10.1016/S01918869(03)00110-7

Olza, I. (2008). De la teoría del vínculo a la neurobiología del apego. Maternidad y ciclo vital de la mujer. 121-125.

Oliva, A. (2004). Estado Actual de la Teoría del Apego. Revista de Psiquiatría y Psicología del Niño y Adolescente, 4(1), 65-81.

Oliva, A. (2011). Apego en la adolescencia. Acción psicológica, 8(2), 55-65. Recuperado de http:// revistas.uned.es/index.php/accionpsicologica/ article/viewFile/190/144

Ortiz-Granja, D.,Acosta-Rodas, P., Lepe-Martínez, M., Del Valle, M.,\& Ramos-Galarza, C. (2019) Escala reducida para valorar el sentido de coherencia: SOC-15. Revista Ecuatoriana de Neurología. Quito-Ecuador: Pontificia Universidad Católica del Ecuador.

Panksepp, J. (1998). Affective Neuroscience: the foundation of human and animal emotions. New York: Oxford University Press.

Pinedo, J., \& Santelices, M. P. (2006). Apego adulto: Los Modelos Operantes Internos y la Teoría de la Mente. Terapia Psicológica, 24(2), 201-209.

Pinto-Cortez, C., Beyzaga, M., Cantero, M. F., Oviedo, X., \& Vergara, V. (2018). Apego y psicopatología en adolescentes del Norte de Chile. Revista de Psicología Clínica con Niños y Adolescentes, 5(3), 23-29. https://doi.org/10.21134/ rpcna.2018.05.3.3

Rasmussen, P. D., Bilenberg, N., Shmueli-Goetz, Y., Simonsen, E., \& Bo Bojesen, A. S. (2019). Attachment representations in Mother and their children diagnosed with ADHD: distribution, transmission and impact on treatment outcome. Journal of Child and Family Studies. No. 28., 1018-1028. doi: 10.1007/s10826-019-01344-5.

Salvador, M. (2013). La sabiduría del cerebro profundo. Bonding, 14(1), 1-14. 
Schore, A. (1994). Affect regulation and the origin of the self: the neurobiology of emotional development. Hilldale, NJ.: Lawrence Eribaum.

Schore, A. (2003). Affect Dysregulation and Disorders of the self. New York: Norton.

Siegel, D. (1999). The Developing Mind: Toward a Neurobiology of interpersonal experience. New York: Guilford.

Soon, C., Braas, M., Haynes, J.D. (2008). Unconscious determinants of free decisions in the human brain. Nature Neuroscience. 543-545.

Sperling, M. B., \& Berman, W. H. (Eds.). (1994). Attachment in adults: Clinical and developmental perspectives. Guilford Press.
Spielmann, S., MacDonald, G., Maxwell, J., Joel, S., Peragine, D., Muise, A., (2013). Settling for less out of fear of being single. Journal of Personality and Social Psychology , 105 (6), 1049-1073. doi: $10.1037 / \mathrm{a} 0034628$

Tulving, E. (1972). Episodic and semantic memory. New York: Academic Press.

Valle, L. \& La Moral, M., (2018): Dependencia emocional y estilo de apego adulto en las relaciones de noviazgo en jóvenes españoles. Rev. iberoam.psicol. salud 9 (1), p. 27. doi: 10.23923/j. rips.2018.01.013

Fecha de recepción: 6 de agosto 2019

Fecha de aceptación: 9 de setiembre 2019 